\title{
Orbits Theory. A Complete Proof of the Collatz Conjecture
}

\author{
PhD. Jorge Crespo Álvarez \\ Prof. Applied Mathematics at Universidad Europea del Atlántico \\ jorge.crespo@uneatlantico.es
}

\begin{abstract}
:
Collatz's Conjecture, enunciated in 1937, remains, to this day, one of the simplest problems to enunciate and yet one of the most difficult to solve. In this work a complete proof of the Collatz Conjecture is presented. The solution assumes as hypothesis that Collatz's Conjecture is a consequence. We start with the inverse of the Collatz's function and manipulate it. We found that every natural number $n_{i} \in \mathbb{N}$ can be calculated starting from 1 , using the function $n_{i}=\left(2^{i-\Omega}-C\right) / 3^{\Omega}$, where: $i \geq 0$ represents the number of steps (operations of multiplications by two subtractions of one and divisions by three) needed to get from 1 to $n_{i}, \Omega \geq 0$ represents the number of multiplications by three required and $0 \leq C \leq 2^{i-\left\lfloor\frac{i}{3}\right\rfloor}-2^{(i \bmod 3)} 3^{\left\lfloor\frac{i}{3}\right\rfloor}$ is an accumulative constant that takes into account the order in which the operations of multiplication and division have been performed.

Reversing the inversion, we have obtained the function: $\left(3^{\Omega} n_{i}+C\right) / 2^{i-\Omega}=1$ that proves that Collatz Conjecture it's a consequence of the above and also proofs that Collatz Conjecture it's true since $\left(3^{\Omega} n_{i}+C\right) / 2^{i-\Omega}$ is the recursive form of the Collatz's function. The works has shown that for each $n_{i, j} \in O_{i}$ is meets that $2^{(i \bmod 3)} \leq n_{i, j} \leq 2^{i}$. Moreover, it's also proofed that there is no loop other than $1,2,4,1 \ldots$
\end{abstract}

\section{Introduction:}

Let the following operation applicable to any natural number:

$$
f(n)=\left\{\begin{array}{cc}
\frac{n}{2} ; & n \text { even } \\
3 n+1 ; & n \text { odd }
\end{array}\right.
$$

The Collatz Conjecture states that, regardless the number $n$ that is chosen, after applying the function $f(n)$ a certain number of steps, the result always tends to 1 , being a single loop of $4,2,1$.

Previous works so far had not been able to prove the Collatz's Conjecture. Different approaches has been taken: Reduced Collatz Conjecture [1], Clustering [2], Orbits [3], Convergence [4], etc. On the other hand, authors as [5] and [6] had worked in the reverse form of the conjecture.

In our current understanding of positive natural numbers, we assume that the way to obtain the next natural number in the sequence is $n_{i+1}=n_{i}+1$.

Starting with $n_{0}=1$, we can generate all natural numbers using the above formula. So, the question to answer is whether this is the only possible way to generate all the natural numbers.

This paper explores the possibility that all natural numbers can be expressed according to:

$$
n_{i}=\frac{2^{i-\Omega} * 1-C}{3^{\Omega}}, \forall n_{i} \in \mathbb{N}^{+} ; 2^{i-\Omega} * 1-C \equiv 0(\bmod 3)
$$

where: $i \geq 0$ represents the number of steps (operations of multiplications by two subtractions of one and divisions by three) needed to get from 1 to $n_{i}, \Omega \geq 0$ represents the number of multiplications by three required and $0 \leq C \leq 2^{i-\left\lfloor\frac{i}{3}\right\rfloor}-2^{(i \bmod 3)} 3^{\left\lfloor\frac{i}{3}\right\rfloor}$ is an accumulative constant that takes into account the order in which the operations of multiplication and division have been performed.

If we clear the 1 in the above equation, we have:

$$
\frac{3^{\Omega} n_{i}+C}{2^{i-\Omega}}=1, \forall n_{i} \in \mathbb{N}^{+} ; 3^{\Omega} n_{i}+C \equiv 0(\bmod 2)
$$


This implies that any natural number could be reduced to 1 after a given number of multiplications by 3 and sum of 1 and divisions by 2. This is precisely what Collatz's Conjecture states.

So, for this demonstration we are going to assume as hypothesis that Collatz's Conjecture is a consequence. We will focus our efforts to demonstrate that that all natural numbers can be expressed according to:

$$
n_{i}=\frac{2^{i-\Omega}-C}{3^{\Omega}}, \forall n_{i} \in \mathbb{N}^{+} ; 2^{i-\Omega} * 1-C \equiv 0(\bmod 3)
$$

\section{Theorem 1. (Paucres-Collatz Theorem):}

Let $\mathrm{N}$ be a positive natural number $\left(N \in \mathbb{N}^{+}\right)$in the Orbit $O_{i}$, then $\mathrm{N}$ meets that:

$$
\frac{3^{\Omega} N+C}{2^{i-\Omega}}=1, \forall N \in \mathbb{N}^{+} ; 3^{\Omega} N+C \equiv 0(\bmod 2)
$$

After $\Omega$ multiplications by 3 and sum of $1, i-\Omega$ divisions by 2 where $C=2^{\alpha}\left(2^{\gamma+\theta+\lambda+\cdots+\psi}+\right.$ $\left.2^{\theta+\lambda+\cdots+\psi} 3^{\Omega-\Omega+1}+\cdots+2^{\psi} 3^{\Omega-2}+3^{\Omega-1}\right) ; C \geq 0$ is an accumulation constant that takes into account the number and the order in which the operations have been performed.

Proof:

\section{1. (Part I): Preliminaries}

Let's take the following function defined for natural numbers:

$$
f(n)=\left\{\begin{array}{cc}
\frac{n}{2} ; & n \text { even } \\
3 n+1 ; & n \text { odd }
\end{array}\right.
$$

Let's calculate the inverse of the function:

Let:

$$
y=\frac{n}{2} ; n \text { even }
$$

Then

$$
y^{-1}=2 n ; \forall n \in \mathbb{N}
$$

Let

$$
y=3 n+1 ; n \text { odd }
$$

Then:

$$
y^{-1}=\frac{n-1}{3} ; \forall n \text { even } \in \mathbb{N} ; n-1 \equiv 0(\bmod 3)
$$

Therefore, the inversion of Collatz's Conjecture implies that:

$$
f(n)=\left\{\begin{array}{cc}
2 n ; & \forall n \in \mathbb{N}^{+} \\
\frac{n-1}{3} ; & \forall n \text { even } \in \mathbb{N} ; n-1 \equiv 0(\bmod 3)
\end{array}\right.
$$

Proposition:

$$
f\left(n_{i}\right)=\left\{\begin{array}{cc}
2 n_{i} ; & \forall n_{i} \in \mathbb{N}^{+} \\
\frac{n_{i}-1}{3} ; & \forall n_{i} \text { even } \in \mathbb{N} ; n_{i}-1 \equiv 0(\bmod 3)
\end{array}\right.
$$

Or in its recursive form:

$$
n_{i}=\frac{2^{i-\Omega}-C}{3^{\Omega}}, \forall n_{i} \in \mathbb{N}^{+} ; 2^{i-\Omega} * 1-C \equiv 0(\bmod 3)
$$

Can be used to calculate any natural number $n_{i}$ starting from 1 . 


\section{2. (Part II). Definition of Orbit, Multifunction and Path}

Let define as Paucres's Orbit $i$ and denoted as $O_{i}$ the set of all natural numbers that share the condition of being $i$ steps (operations) from 1 . This implies that:

$$
\begin{gathered}
1 \in O_{0} \\
2 \in O_{1} \\
4 \in O_{2} \\
(8,1) \in O_{3} \\
(16,2) \in O_{4} \\
(32,5,4) \in O_{5}
\end{gathered}
$$

And so on.

Let define as $n_{i, j}$ each of the natural numbers that belongs to the Orbit $O_{i}$

$$
n_{i, j} \in O_{i}
$$

Let define a Multifunction $M\left(O_{i}\right)$ that allows to calculate the set of the $n_{i+1, j}$ positive natural numbers that belongs to the Orbit $O_{i+1}$ from all the $n_{i, j}$ numbers of $O_{i}$ and therefore $n_{i+1, j}$ are at $i+1$ steps from 1 .

$$
O_{i+1}=M\left(O_{i}\right)
$$

Therefore:

$$
M\left(O_{i}\right)\left\{\begin{array}{c}
n_{i+1, j}=2 n_{i, j} \forall n_{i, j} \in O_{i} \\
n_{i+1, j}=\frac{n_{i, j}-1}{3} \forall n_{i, j} \text { even } \in O_{i} ; n_{i, j}-1 \equiv 0(\bmod 3)
\end{array}\right.
$$

According to the above:

- $\quad$ Every ancestor $n_{i, j}$ has an even $n_{i+1, j}$ successor

- $\quad$ Every ancestor $n_{i, j}$, has, at most, two $n_{i+1, j}$ successors (one even and one odd)

Let's define as Path to the succession of terms $n_{i, j}, n_{i-1, j}, n_{i-2, j}, \ldots, 1$ required to calculate the term $n_{i+1, j}$ applying the Multifunction $M\left(O_{i}\right)$.

2.3. (Part III). Lemma 1.: Any positive natural number can be expressed in the form $2^{\alpha} \beta$ where $\beta$ is an odd natural number greater than or equal to 1 and $\alpha$ is a natural number greater than or equal to 0 .

Proof:

Let $J_{0}$ an even natural number, then it can be expressed as:

$$
J_{0}=2 * J_{1}
$$

Where $J_{1}=\frac{J_{0}}{2}$

If $J_{1}$ even, then it can be expressed as:

$$
J_{1}=2 * J_{2}
$$

Where $J_{2}=\frac{J_{1}}{2}$

This implies:

$$
J_{0}=2 * 2 * J_{2}=2^{2} * J_{2}
$$

Repeating this process until we find an odd $J_{n}$ we have:

$$
J_{0}=2^{n} * J_{n}
$$


Thus, it is true that any even number can be expressed as $2^{\alpha} \beta$, where $\beta$ is an odd natural number greater than or equal to 1 and $\alpha$ an natural number greater than or equals to 1 .

Let $J_{0}$ an odd natural number, then it can be expressed as:

$$
J_{0}=2^{0} * J_{0}
$$

Thus, it is true that any odd number can be expressed as $2^{\alpha} \beta$ where $\beta$ is an odd natural number greater than or equal to 1 and $\alpha$ an natural number equals to 0 .

Thus, it is true that any natural number can be expressed as $2^{\alpha} \beta$ where $\beta$ is an odd natural number greater than or equal to 1 and $\alpha$ is a natural number greater than or equal to 0 .

\section{4. (Part IV). Lemma 2.: Every odd number obtained by the multifunction $M\left(O_{i}\right)$ depends, indirectly,} on an odd ancestor

Proof:

Any odd number $n_{i+1, j}$ obtained from the multifunction $M\left(O_{i}\right)$ depends on an even $n_{i, j}$ ancestor that meets the condition:

$$
n_{i+1, j}=\frac{n_{i, j}-1}{3} \forall n_{i, j} \text { even } \in O_{i} ; n_{i, j}-1 \equiv 0(\bmod 3)
$$

But, since $n_{i, j}$ is even, it can be written as $2^{\alpha} \beta$. Therefore, the above expression can be rewritten as:

$$
n_{i+1, j}=\frac{2^{\alpha} \beta-1}{3} ; 2^{\alpha} \beta-1 \equiv 0(\bmod 3)
$$

But $\beta$ is an odd natural number greater than or equal to 1 , so every odd number depends, indirectly, on an odd number.

2.5. (Part V). Lemma 3.: 1 is the only odd natural number that depends on itself after a single division by three.

Proof:

Let

$$
\beta=\frac{2^{\alpha} \beta-1}{3}
$$

Solving for $\beta$ from the equation above we have:

$$
\beta=\frac{1}{2^{\alpha}-3}
$$

Since $\beta$ is an odd natural number greater than or equal to 1 , it has to be met that:

$$
\begin{aligned}
& 2^{\alpha}-3>0 \\
& 2^{\alpha}-3 \leq 1
\end{aligned}
$$

Solving the previous inequalities for $\alpha$ we have:

$$
\begin{aligned}
& \alpha>\log _{2} 3 \\
& \alpha \leq \log _{2} 4
\end{aligned}
$$

Thus:

$$
\alpha=2
$$

And therefore:

$$
\beta=\frac{1}{2^{2}-3}=1
$$


Therefore, the only odd natural number that depends on itself after a division by three is 1 .

In other words, 1 is the only odd natural number with the ability to self-generate after a division by three.

\section{Lemma 3.}

Let $\beta$ be an odd natural number, it is satisfied that $\beta=1$ is the only odd that meets the condition:

$$
\beta=\frac{2^{2} \beta-1}{3}
$$

2.6. (Part VI). Lemma 4.: $n_{i, j}=\frac{2^{i-\Omega}-C}{3^{\Omega}} ; 2^{i-\Omega}-C \equiv 0(\bmod 3)$ is a general expression for $M\left(O_{i}\right)$.

Proof:

Denote $\mathrm{A}$ as the number of multiplications by two, denote $\Omega$ as the number of divisions by three required to obtain a number from 1 .

Let's define a Paucres's root as:

$$
2^{0}=1
$$

Let's define as a Paucres's even to any even natural number that can be obtained from the root according to:

$$
n_{\mathrm{A}, j}=\frac{2^{\mathrm{A}} * 2^{0}-0}{1}
$$

Where $A=i$ is the index of the Orbit of the even generated. $A \geq 1$ since $2^{1}=2$ is the first even positive natural number.

Let's define as a Paucres's odd to any odd natural number obtained from a Paucres's even as:

$$
n_{\mathrm{A}+\Omega, j}=\frac{\frac{2^{\mathrm{A}} * 2^{0}-0}{1}-3^{0}}{3^{1}} ; 2^{A}-1 \equiv 0(\bmod 3)
$$

Where $\mathrm{A}+1=\mathrm{i}$ is the index of the Orbit of the odd generated. A (even) $\geq 2$ since $2^{2}=4$ is the first even natural number that generates an odd natural number. Let's denote $1=\Omega$ because there is a single division by three. Thus, $A+\Omega=i$ is the Orbit of the odd generated. Then:

$$
n_{\mathrm{A}+\Omega, j}=\frac{\frac{2^{\mathrm{A}} * 2^{0}-0}{1}-3^{0}}{3^{1}}=\frac{2^{\mathrm{A}} * 2^{0}-0-3^{\Omega-1}}{3^{\Omega}}=\frac{2^{\mathrm{A}} * 2^{0}-3^{\Omega-1}}{3^{\Omega}}=\frac{2^{\mathrm{A}}-3^{\Omega-1}}{3^{\Omega}} ; 2^{A}-3^{\Omega-1} \equiv 0(\bmod 3)
$$

Let's take a $n_{\mathrm{A}+\gamma+\Omega+1, j}=\frac{2^{\gamma}\left(n_{\mathrm{A}+\Omega, j}\right)-1}{3}$ odd natural number obtained from $n_{\mathrm{A}+\Omega, j}$ after a given $\alpha$ number of multiplications by 2 , a subtraction of 1 and a division by three.

Substituting $n_{\mathrm{A}+\Omega, j}=\frac{2^{\mathrm{A}}-3^{\Omega-1}}{3^{\Omega}}$ into $n_{\mathrm{A}+\gamma+\Omega+1, j}=\frac{2^{\gamma}\left(n_{\mathrm{A}+\Omega, j}\right)-1}{3}$ we have:

$$
n_{\mathrm{A}+\gamma+\Omega+1, j}=\frac{2^{\gamma} \frac{2^{\mathrm{A}}-3^{\Omega-1}}{3^{\Omega}}-1}{3}=\frac{2^{A+\gamma}-\left(2^{\gamma}+3^{\Omega}\right)}{3^{\Omega+1}} ; 2^{A+\gamma}-\left(2^{\gamma}+3^{\Omega}\right) \equiv 0(\bmod 3)
$$

Updating the value of $\mathrm{A}+\gamma=A$ and $\Omega+1=\Omega$, the above expression can be written as:

$$
n_{\mathrm{A}+\Omega, j}=\frac{2^{A}-\left(2^{\gamma}+3^{\Omega-1}\right)}{3^{\Omega}} ; 2^{A}-\left(2^{\gamma}+3^{\Omega}\right) \equiv 0(\bmod 3)
$$

Let's call $2^{\gamma}+3^{\Omega-1}=C$, then $C$ is an accumulation constant that takes into account the number operations of multiplication performed before dividing by three:

$$
n_{\mathrm{A}+\Omega, j}=\frac{2^{A}-C}{3^{\Omega}} ; 2^{A}-C \equiv 0(\bmod 3)
$$

Now let's define a new odd that depends on the updated $n_{\mathrm{A}+\Omega, j}$ according to: 


$$
n_{\mathrm{A}+\theta+\Omega+1, j}=\frac{2^{\theta}\left(n_{\mathrm{A}+\Omega, j}\right)-1}{3}
$$

Substituting, we have:

$$
\begin{gathered}
n_{\mathrm{A}+\theta+\Omega+1, j}=\frac{2^{\theta}\left(\frac{2^{A}-\left(2^{\gamma}+3^{\Omega-1}\right)}{3^{\Omega}}\right)-1}{3}=\frac{2^{A+\theta}-2^{\theta}\left(2^{\gamma}+3^{\Omega-1}\right)-3^{\Omega}}{3^{\Omega+1}} \\
n_{\mathrm{A}+\theta+\Omega+1, j}=\frac{2^{A+\theta}-2^{\gamma+\theta}-2^{\theta} 3^{\Omega-1}-3^{\Omega}}{3^{\Omega+1}} ; 2^{A+\theta}-2^{\gamma+\theta}-2^{\theta} 3^{\Omega-1}-3^{\Omega} \equiv 0(\bmod 3)
\end{gathered}
$$

Updating the value of $\mathrm{A}+\theta=A$ and $\Omega+1=\Omega$, the above expression can be written as:

$$
n_{\mathrm{A}+\Omega, j}=\frac{2^{\mathrm{A}}-C}{3^{\Omega}} ; 2^{A}-C \equiv 0(\bmod 3)
$$

With

$$
C=2^{\gamma+\theta}+2^{\theta} 3^{\Omega-2}+3^{\Omega-1}
$$

Recursively, then any odd natural number $n_{\mathrm{A}+\Omega, j}$ can be expressed according to:

$$
n_{\mathrm{A}+\Omega, j}=\frac{2^{\mathrm{A}}-C}{3^{\Omega}} ; 2^{A}-C \equiv 0(\bmod 3)
$$

With

$$
C=2^{\gamma+\theta+\cdots+\lambda+\psi}+2^{\theta+\cdots+\lambda} 3^{\Omega-\Omega+1}+\cdots+2^{\theta} 3^{\Omega-2}+3^{\Omega-1}
$$

On the other hand, as all $n_{\mathrm{A}+\Omega, j}$ has been obtained after applying the multifunction $M\left(O_{i}\right)$, this implies that the sum of the number of multiplications by two plus the number of divisions by three has to be equal to the index $i$ of the Orbit $O_{i}$.

This implies that $\mathrm{A}+\Omega=i$, thus, $\mathrm{A}=i-\Omega$.

In this way, the above expression can be written as:

$$
n_{i, j}=\frac{2^{i-\Omega}-C}{3^{\Omega}} ; 2^{i-\Omega}-C \equiv 0(\bmod 3)
$$

Which is a valid expression to obtain all odd natural numbers for any Orbit $O_{i}$.

Moreover, if $n_{i, j}$ is odd, then it can be used to generate an even number according to $2^{\alpha} \beta$, then:

$$
\begin{gathered}
n_{i+\alpha, j}=2^{\alpha} \frac{2^{i-\Omega}-C}{3^{\Omega}} ; 2^{i-\Omega}-C \equiv 0(\bmod 3) \\
n_{i+\alpha, j}=\frac{2^{i+\alpha-\Omega}-2^{\alpha} C}{3^{\Omega}} ; 2^{i+\alpha-\Omega}-2^{\alpha} C \equiv 0(\bmod 3)
\end{gathered}
$$

Rewriting the above expression to refer to $n_{i, j}$ we have:

$$
n_{i, j}=\frac{2^{i-\Omega}-2^{\alpha} C}{3^{\Omega}} ; 2^{i-\Omega}-2^{\alpha} C \equiv 0(\bmod 3)
$$

Updating $2^{\alpha} C=C$ we have:

$$
n_{i, j}=\frac{2^{i-\Omega}-C}{3^{\Omega}} ; 2^{i-\Omega}-C \equiv 0(\bmod 3)
$$

Therefore, the expression $n_{i, j}=\frac{2^{i-\Omega}-C}{3^{\Omega}} ; 2^{i-\Omega}-C \equiv 0(\bmod 3)$ is valid to represent any positive natural number obtained from $M\left(O_{i}\right)$.

Where:

$i$ represents the Orbit of $n_{i, j}$

$\Omega$ represents the number of divisions by three needed to obtain $n_{i, j}$ 
$C=2^{\alpha}\left(2^{\gamma+\theta+\lambda+\cdots+\psi}+2^{\theta+\lambda+\cdots+\psi} 3^{\Omega-\Omega+1}+\cdots+2^{\psi} 3^{\Omega-2}+3^{\Omega-1}\right)$ is an integer accumulation constant greater than or equal to zero that takes into account the number and order in which the operations of multiplication by 2 and subtraction of 1 and division by three have been performed.

2.7. (Part VII). Lemma 5.: $n_{i, j}=\frac{2^{i-\Omega}-C}{3^{\Omega}} ; 2^{i-\Omega}-C \equiv 0(\bmod 3)$ is bounded from above. Proof:

Let

$$
n_{i, j}=\frac{2^{i-\Omega}-C}{3^{\Omega}}
$$

Then

$$
n_{i, j}=\frac{2^{i-\Omega}-C}{3^{\Omega}} \leq \frac{2^{i-\Omega}}{3^{\Omega}} \leq \frac{2^{i}}{3^{\Omega}} \leq 2^{i}
$$

Therefore, $n_{i, j} \leq 2^{i}$ and then $\operatorname{Max}\left(n_{i, j}\right)=2^{i}$

Solving for $i$ we have:

$$
i=\log _{2} n_{i, j}
$$

But $i$ must be an integer greater than or equal to 0 , then:

$$
i=\left\lfloor\log _{2} n_{i, j}\right\rfloor
$$

The above proof has great connotation for the demonstration of the Collatz Conjecture, since it implies that, for any positive natural number $N$ it is possible to find an $O_{i}$, from which any $n_{i, j} \in O_{i}$ is less than or equal to $\mathrm{N}$.

$$
\forall N \in \mathbb{N}^{+} \exists i ; \forall n_{i, j} \in O_{i}, n_{i, j} \leq N
$$

Where:

$$
i=\left\lfloor\log _{2} N\right\rfloor
$$

2.8. (Part VIII). Lemma 6.: $n_{i, j}=\frac{2^{i-\Omega}-C}{3^{\Omega}}$ is bounded from below.

Proof.

For an Orbit $i$, with $i \geq 0$ and a number $\Omega$ of divisions by three it is satisfied that:

$$
n_{i, j}=\frac{2^{i-\Omega}-C}{3^{\Omega}}
$$

According to the constraints in the statement of the problem, every $n_{i, j}$ is a positive natural number, therefore, it has to meet that:

$$
n_{i, j}=\frac{2^{i-\Omega}-C}{3^{\Omega}} \geq 1
$$

This implies:

$$
\begin{aligned}
& \operatorname{Min}\left(n_{i, j}\right) \geq 1 \\
& \operatorname{Min}\left(n_{i, j}\right)=1
\end{aligned}
$$

The constraints of the problem cause the function to be bounded from below.

However, it was shown that the value of 1 is reached infinite times in the loop 1, 2, 4, 1.... However, only for some values of $i$ it's meet that $n_{i, j}=1$. 
This implies that there must be a function that generates the appropriate succession of terms of the 1, 2, 4, 1 ... loop for each value of $i$.

For fixed values of $i, \Omega$ it has to be meet that:

$$
\operatorname{Min}\left(n_{i, j}\right)=\frac{2^{i-\Omega}-\operatorname{Max}(C)}{3^{\Omega}}
$$

Previously it has been shown that:

$$
1=\frac{2^{2}-1}{3}=\frac{4-1}{3}
$$

Rewriting the above equation in the form of the general equation we have:

$$
1=\frac{2^{i-\Omega}-\operatorname{Max}(C)}{3^{\Omega}}=\frac{2^{3-1}-1}{3^{1}}=\frac{2^{2}-1}{3}
$$

Multiplying by 2 on both sides of the equal sign we have:

$$
2=\frac{2^{2+1}-2 * 1}{3^{1}}=\frac{2^{3}-2}{3}
$$

Multiplying again by 2 on both sides of the equal sign we have:

$$
4=\frac{2^{3+1}-2 * 2 * 1}{3^{1}}=\frac{2^{4}-4}{3}
$$

But 4 can be used to generate 1 , subtracting 1 and dividing by three. Therefore:

$$
1=\frac{\frac{2^{4}-4}{3}-1}{3^{1}}=\frac{2^{4}-7}{3^{2}}
$$

This implies that, recursively, all the terms of $\operatorname{Max}(C)$ can be calculated for a given values of $i, \Omega$.

We will proceed to establish a calculation method for $\Omega$ as a function of $i$ for these conditions.

The loop 1, 2, 4, 1... has 3 steps (two multiplications by 2 and a division by three). After these steps, the value of $\Omega$ is increased by 1 . Let's then define a way to calculate $\Omega$ as a function of $i$.

Let:

$$
\Omega=\left\lfloor\frac{i}{3}\right\rfloor
$$

We will now proceed to find an expression for $\operatorname{Max}(C)$ for each values of $i$ :

$$
\frac{2^{i-\left\lfloor\frac{i}{3}\right\rfloor}-\operatorname{Max}(C)}{3^{\left\lfloor\frac{i}{3}\right\rfloor}}=1
$$

Clearing $\operatorname{Max}(C)$ we have:

$$
2^{i-\left\lfloor\frac{i}{3}\right\rfloor}-3^{\left\lfloor\frac{i}{3}\right\rfloor}=\operatorname{Max}(C)
$$

Taking a common factor of $2^{0}$ in the member of the left we have:

$$
2^{0}\left(2^{i-\left\lfloor\frac{i}{3}\right\rfloor}-3^{\left\lfloor\frac{i}{3}\right\rfloor}\right)=\operatorname{Max}(C)
$$

The above equation allows to calculate the values of $\operatorname{Max}(C)$ for:

$$
i=0+3 k ; k \in \mathbb{N}, k \geq 0
$$

For the second case we have:

$$
\frac{2^{i-\left\lfloor\frac{i}{3}\right\rfloor}-\operatorname{Max}(C)}{3^{\left\lfloor\frac{i}{3}\right\rfloor}}=2
$$

Clearing $\operatorname{Max}(C)$ we have: 


$$
2^{i-\left\lfloor\frac{i}{3}\right\rfloor}-2 * 3^{\left\lfloor\frac{i}{3}\right\rfloor}=\operatorname{Max}(C)
$$

Taking a common factor of $2^{1}$ in the member of the left we have:

$$
2^{1}\left(2^{i-1-\left\lfloor\frac{i}{3}\right\rfloor}-3^{\left\lfloor\frac{i}{3}\right\rfloor}\right)=\operatorname{Max}(C)
$$

The above equation allows to calculate the values of $\operatorname{Max}(C)$ for:

$$
i=1+3 k ; k \in \mathbb{N}, k \geq 0
$$

For the third case we have:

$$
\frac{2^{i-\left\lfloor\frac{i}{3}\right\rfloor}-\operatorname{Max}(C)}{\left.3^{\lfloor} \frac{i}{3}\right\rfloor}=4
$$

Clearing $\operatorname{Max}(C)$ we have:

$$
2^{i-\left\lfloor\frac{i}{3}\right\rfloor}-4 * 3^{\left\lfloor\frac{i}{3}\right\rfloor}=\operatorname{Max}(C)
$$

Taking a common factor of $2^{2}$ in the member of the left we have:

$$
2^{2}\left(2^{i-2-\left\lfloor\frac{i}{3}\right\rfloor}-3^{\left\lfloor\frac{i}{3}\right\rfloor}\right)=\operatorname{Max}(C)
$$

The above equation allows to calculate the values of $\operatorname{Max}(C)$ for:

$$
i=2+3 k ; k \in \mathbb{N}, k \geq 0
$$

Now we will proceed to find a general expression for $\operatorname{Max}(C) \forall i \geq 0$ by combining the above three equations:

$$
\begin{gathered}
2^{(i \bmod 3)}\left(2^{i-(i \bmod 3)-\left\lfloor\frac{i}{3}\right\rfloor}-3^{\left\lfloor\frac{i}{3}\right\rfloor}\right)=\operatorname{Max}(C) \\
2^{i-\left\lfloor\frac{i}{3}\right\rfloor}-2^{(i \bmod 3)} 3^{\left\lfloor\frac{i}{3}\right\rfloor}=\operatorname{Max}(C)
\end{gathered}
$$

In such a way that:

$$
\begin{gathered}
\operatorname{Min}\left(n_{i, j}\right)=\frac{2^{i-\left\lfloor\frac{i}{3}\right\rfloor}-\left(2^{i-\left\lfloor\frac{i}{3}\right\rfloor}-2^{(i \bmod 3)} 3^{\left\lfloor\frac{i}{3}\right\rfloor}\right)}{3^{\left\lfloor\frac{i}{3}\right\rfloor}} \\
\operatorname{Min}\left(n_{i, j}\right)=\frac{2^{(i \bmod 3)} 3^{\left.\frac{i}{3}\right\rfloor}}{\left.3^{\left\lfloor\frac{i}{3}\right.}\right\rfloor} \\
\operatorname{Min}\left(n_{i, j}\right)=2^{(i \bmod 3)}
\end{gathered}
$$

Then, $\frac{2^{i-\Omega}-C}{3^{\Omega}}$ is bounded:

$$
2^{(i \bmod 3)} \leq \frac{2^{i-\Omega}-C}{3^{\Omega}} \leq 2^{i}
$$

With:

$$
0 \leq \Omega \leq\left\lfloor\frac{i}{3}\right\rfloor
$$

And:

$$
\left.0 \leq C \leq 2^{i-\left\lfloor\frac{i}{3}\right\rfloor}-2^{(i \bmod 3)} 3^{3^{i}}\right\rfloor
$$

2.9. (Part IX). Lemma 7.: $\lim _{i \rightarrow 0} \frac{2^{i-\Omega}-C}{3^{\Omega}}=1$

Proof:

Let's define the following functions in $\mathbb{R}$ : 


$$
\begin{gathered}
f(x)=2^{x} \\
g(x)=2^{(x \bmod 3)} \\
h(x)=\frac{2^{x-a}-C}{3^{a}}, 0 \leq a \leq\left\lfloor\frac{x}{3}\right\rfloor, 0 \leq C \leq 2^{x-\left\lfloor\frac{x}{3}\right\rfloor}-2^{(x \bmod 3)} 3^{\left\lfloor\frac{x}{3}\right\rfloor}
\end{gathered}
$$

Let's calculate the limit when $x \rightarrow 0$ for the three functions:

$$
\begin{gathered}
\lim _{x \rightarrow 0} 2^{x}=2^{0}=1 \\
\lim _{x \rightarrow 0} 2^{(x \bmod 3)}=2^{0}=1
\end{gathered}
$$

Applying the intermediate function theorem, then:

$$
\lim _{x \rightarrow 0} \frac{2^{x-a}-C}{3^{a}}=1
$$

Thus:

$$
\lim _{i \rightarrow 0} \frac{2^{i-\Omega}-C}{3^{\Omega}}=\lim _{i \rightarrow 0} n_{i, j}=1
$$

The result of the limit above shows that, for all $n_{i, j} \in O_{i}$, there is a Path $n_{i-1, j}, n_{i-2, j}, \ldots n_{i-(i-3), j}, 4,2,1$ that converges to 1 .

2.10 (Part X): Lemma 8.: All positive natural numbers can be expressed as $N=\frac{2^{i-\Omega_{* 1}-C}}{3^{\Omega}}, \forall N \in \mathbb{N}^{+}$

Proof:

Let's suppose that:

$$
N \neq \frac{2^{i-\Omega} * 1-C}{3^{\Omega}}
$$

With:

$$
0 \leq \Omega \leq\left\lfloor\frac{i}{3}\right\rfloor
$$

And:

$$
0 \leq C \leq 2^{i-\left\lfloor\frac{i}{3}\right\rfloor}-2^{(i \bmod 3)} 3^{\left\lfloor\frac{i}{3}\right\rfloor}
$$

Clearing for $i$ we have:

$$
i \neq \log _{2}\left(6^{\Omega} N+2^{\Omega} C\right)
$$

Let's define:

$$
y=f(x)=\log _{2}\left(6^{\Omega} x+2^{\Omega} C\right) ; x \geq 1
$$

Under this conditions, it's meet that:

$$
6^{\Omega} x+2^{\Omega} C \geq 1, \forall x \geq 1 ; \Omega, C \geq 0
$$

As $f(x)$ is a logarithmic function then its domain is:

$$
D=\left\{(x) \mid 6^{\Omega} x+2^{\Omega} C \geq 1\right\}=\{x \in \mathbb{R} ; x \geq 1\}
$$

In addition, all logarithmic functions are continuous throughout their domain, then $f(x)$ is continuous throughout its domain.

The range of $f(x)$ is:

$$
\operatorname{Range}(f(x))=\{y \in \mathbb{R} ; y \geq 0\}=[0, \infty)
$$

As $f(x)$ is a logarithmic function, then the range of $f(x)$ is also continuous. 
Then:

$$
y \neq \log _{2}\left(6^{\Omega} x+2^{\Omega} C\right) \forall y \geq 0
$$

Is a contradiction.

Thus:

$$
i \neq \log _{2}\left(6^{\Omega} N+2^{\Omega} C\right) \forall i \geq 0
$$

Is also a contradiction.

Then:

$$
i=\log _{2}\left(6^{\Omega} N+2^{\Omega} C\right) \forall i \geq 0
$$

This implies that:

$$
\forall N \in \mathbb{N}^{+} \exists \Omega, C \geq 0 ; 6^{\Omega} N+2^{\Omega} C=2^{i}
$$

After the previous proof we can state the following:

\section{Lemma 8.}

Let $\mathrm{N}$ be a positive natural number; then $\mathrm{N}$ meets that:

$$
N=\frac{2^{i-\Omega} * 1-C}{3^{\Omega}}, \forall N \in \mathbb{N}^{+} ; 2^{i-\Omega} * 1-C \equiv 0(\bmod 3)
$$

\subsection{1. (Part XI): Lemma 9.: There is no loop other than 1, 2, 4, $1 \ldots$}

Proof:

If there is a loop other than $1,2,4,1 \ldots$, this implies that a number has the ability to auto-generate after a certain number of multiplications by two, subtractions of one and divisions by three.

Applying the general expression, we will investigate if there is an odd that depends on itself after an $\alpha$ number of steps with $\alpha-\gamma$ multiplications by 2 and $\gamma$ subtractions of 1 and divisions by 3 :

Let:

$$
n_{i, j}=\frac{2^{i-\Omega}-c_{1}}{3^{\Omega}}
$$

Let's define a new $n_{i+\alpha, j}=n_{i, j}$ obtained from $n_{i, j}$ after $\alpha$ number of steps with $\alpha-\gamma$ multiplications by 2 and $\gamma$ subtractions of 1 and divisions by 3 as:

$$
n_{i+\alpha, j}=\frac{2^{\alpha-\gamma} n_{i, j}-c_{2}}{3^{\gamma}}
$$

Substituting $n_{i, j}=\frac{2^{i-\Omega}-c_{1}}{3^{\Omega}}$ into $n_{i+\alpha, j}=\frac{2^{\alpha} n_{i, j}-c_{2}}{3^{\gamma}}$ we have:

$$
n_{i+\alpha, j}=\frac{2^{\alpha-\gamma} \frac{2^{i-\Omega}-c_{1}}{3^{\Omega}}-c_{2}}{3^{\gamma}}
$$

Then:

$$
n_{i+\alpha, j}=\frac{2^{i+\alpha-(\Omega+\gamma)}-C}{3^{\Omega+\gamma}}
$$

Solving for $C$ we have:

$$
C=2^{i+\alpha-(\Omega+\gamma)}-n_{i+\alpha, j} 3^{\Omega+\gamma}
$$

Earlier we showed that:

$$
2^{i-\left\lfloor\frac{i}{3}\right\rfloor}-2^{(i \bmod 3)} 3^{\left\lfloor\frac{i}{3}\right\rfloor}=\operatorname{Max}(C)
$$

For the Orbit $O_{i+\alpha}$ the above equation can be expressed as:

$$
2^{i+\alpha-\left[\frac{i+\alpha}{3}\right\rfloor}-2^{((i+\alpha) \bmod 3)} 3^{\left\lfloor\frac{i+\alpha}{3}\right\rfloor}=\operatorname{Max}(C)
$$


Then:

$$
2^{i+\alpha-(\Omega+\gamma)}-n_{i+\alpha, j} 3^{\Omega+\gamma} \leq 2^{i+\alpha-\left\lfloor\frac{i+\alpha}{3}\right\rfloor}-2^{((i+\alpha) \bmod 3)} 3^{\left\lfloor\frac{i+\alpha}{3}\right\rfloor}
$$

Solving for $n_{i+\alpha, j}$ :

$$
n_{i+\alpha, j} \leq-\frac{2^{i+\alpha-\left\lfloor\frac{i+\alpha}{3}\right\rfloor}-2^{((i+\alpha) \bmod 3)} 3^{\left\lfloor\frac{i+\alpha}{3}\right\rfloor}-2^{i+\alpha-(\Omega+\gamma)}}{3^{\Omega+\gamma}}
$$

Then:

$$
n_{i+\alpha, j} \leq \frac{2^{i+\alpha-(\Omega+\gamma)}-\left(2^{i+\alpha-\left\lfloor\frac{i+\alpha}{3}\right\rfloor}-2^{((i+\alpha) \bmod 3)} 3^{\left\lfloor\frac{i+\alpha}{3}\right\rfloor}\right)}{3^{\Omega+\gamma}}
$$

But:

$$
2^{i+\alpha-\left\lfloor\frac{i+\alpha}{3}\right\rfloor}-2^{((i+\alpha) \bmod 3)} 3^{\left\lfloor\frac{i+\alpha}{3}\right\rfloor}=\operatorname{Max}(C)
$$

Then:

$$
n_{i+\alpha, j} \leq \frac{2^{i+\alpha-(\Omega+\gamma)}-\operatorname{Max}(C)}{3^{\Omega+\gamma}}
$$

But in Lemma 6 we had proofed that:

$$
\operatorname{Min}\left(n_{i, j}\right)=\frac{2^{i-\Omega}-\operatorname{Max}(C)}{3^{\Omega}}
$$

Then for $n_{i+\alpha, j}$ we have:

$$
\operatorname{Min}\left(n_{i+\alpha, j}\right)=\frac{2^{i+\alpha-(\Omega+\gamma)}-\operatorname{Max}(C)}{3^{\Omega+\gamma}}
$$

Thus:

$$
n_{i+\alpha, j} \leq \operatorname{Min}\left(n_{i+\alpha, j}\right)
$$

Then:

$$
\begin{gathered}
n_{i+\alpha, j}=\operatorname{Min}\left(n_{i+\alpha, j}\right) \\
n_{i+\alpha, j}=2^{((i+\alpha) \bmod 3)}=1,2,4,1 \ldots
\end{gathered}
$$

But:

$$
n_{i+\alpha, j}=n_{i, j}
$$

Then:

$$
n_{i, j}=2^{(i \bmod 3)}=1,2,4,1 \ldots
$$

This proof allows to extend the Lemma 3.

\section{Lemma 9.}

Let $N \in \mathbb{N}^{+}$be a positive natural number, then it is satisfied that $N=1, N=2$ and $N=4$ are the only natural numbers that meets the condition:

$$
N=\frac{2^{\alpha-\gamma} N-C}{3^{\gamma}} ; 2^{\alpha-\gamma} N-C \equiv 0(\bmod 3)
$$

after an $\alpha$ number of steps with $\alpha-\gamma$ multiplications by 2 and $\gamma$ subtracts from 1 and divisions by 3

\subsection{2. (Part XII): Final Remarks of the Proof of the Collatz Conjecture:}

After all the previous Lemmas we can now Proof the Collatz Conjecture

In Lemma 8 we have proofed a general expression for $N, \forall N \in \mathbb{N}^{+}$. Then in Lemma 9 we have proofed that there is no loop other than $1,2,4,1 \ldots$ 
Then, let:

$$
N=\frac{2^{i-\Omega} * 1-C}{3^{\Omega}}, \forall N \in \mathbb{N}^{+} ; 2^{i-\Omega} * 1-C \equiv 0(\bmod 3)
$$

Clearing the 1 in the expression above we have:

$$
\frac{3^{\Omega} N+C}{2^{i-\Omega}}=1, \forall N \in \mathbb{N}^{+} ; 3^{\Omega} N+C \equiv 0(\bmod 2)
$$

Which is precisely the proof that Collatz's Conjecture it's true.

\section{References}

[1] W. Ren, "A New Approach on Proving Collatz Conjecture,» Journal of Mathematics, pp. 112, 2019.

[2] J. A. Tenreiro Machado, A. Galhano y D. Cao Labora, «A Clustering Perspective of the Collatz Conjecture, » Mathematics, vol. 9, no 314, pp. 1-14, 2021.

[3] T. Tao, «Almost all orbits of the Collatz map attain almost bounded values,» 18092019. [En línea]. Available: https://arxiv.org/abs/1909.03562.

[4] D. Barina, "Convergence verification of the Collatz problem, " The Journal of Supercomputing, pp. 2681-2688, 2021.

[5] L. Colussi, "The convergence classes of Collatz function," Theoretical Computer Science, vol. 412, no 39, pp. 5409-5419, 2011.

[6] P. C. Hew, «Working in binary protects the repetends of $1 / 3 \mathrm{~h}$ : Comment on Colussi's 'The convergence classes of Collatz function', " Theoretical Computer Science, vol. 618, pp. 135141, 2016. 